\title{
Adaptation and validation of the Parent-Child Conflict Tactics Scale for use in Sri Lanka
}

\author{
Piyanjali de Zoysa ${ }^{1}$ Lalini Rajapakse ${ }^{2}$ and Peter A Newcombe ${ }^{3}$ \\ (Index words: Non-violent discipline, physical abuse, psychological aggression)
}

\begin{abstract}
Objective The purpose of the study was to validate the Parent-Child Conflict Tactics Scale (CTSPC) in Sri Lanka, for use with 12-year old children.

Design A Delphi Process determined the consensual and content validity of the CTSPC. Test-retest reliability and internal consistency were determined by a large and a small group administration.
\end{abstract}

Setting Professionals from the social and behavioural sciences were the judges in the Delphi Process. Conveniently located schools in the Gampaha District were chosen for determining the test-retest reliability and internal consistency.

Measurements This included original CTSPC and its Sinhala translation, a structured interview schedule and a focus group guide.

Results The Sinhala version of the CTSPC showed adequate consensual and content validity. Its test-retest reliability and internal consistency were satisfactory. The instrument is best administered in small rather than in large groups of children.

Conclusion The Sinhala version of the CTSPC is appropriate to be used with 12-year old Sinhala speaking schoolchildren in Sri Lanka.

\section{Introduction}

Concern about the violence in the name of discipline has been growing among professional and lay communities in the country [1]. Its adverse consequences are well documented [2]. However, reliable information on parental disciplinary practices is not available in Sri Lanka [1]. Such statistics are necessary to design culturally relevant preventive intervention programmes to combat violence against children [3]. One of the main reasons for the lack of such information is the dearth of culturally valid instruments to measure such practices.

Several instruments are available that investigate disciplinary strategies [4-6]. The Parent-Child Conflict Tactics Scale (CTSPC) [7], unlike these others, assesses both violent and non-violent techniques. It has shown adequate validity and internal consistency (in its country of origin) and has been adapted and used in many cultural settings [3]. It provides information on four disciplinary categories: (i) non-violence, (ii) psychological aggression, (iii) corporal punishment, and (iv) physical abuse.

Instruments that are based on sound theoretical underpinnings and show adequate validity and reliability in their country of origin, should be appropriately used after cultural validation, to suit different cultural settings $[8,9]$. Such cultural validation of the CTSPC was the goal of the present study.

The common practice in validation is to compare the instrument with the gold standard (i.e. criterion validation). However, in this study, a gold standard was unavailable. This paper reports a validation method that may be used in the absence of a gold standard.

\section{Method}

\section{Translation and pre-tests}

Permission to validate the CTSPC to Sinhala was obtained from its authors. A professional translator translated the instrument to Sinhala, and a second translator translated the Sinhala version back to English. To ascertain the conceptual equivalency of the translations [10], the original CTSPC, its Sinhala version and the translated (from Sinhala to) English version were presented to a bilingual professional from the behavioural sciences to determine any discrepancy. The Sinhala items that did not correspond with the original instrument were corrected.

The first pre-test of the CTSPC's Sinhala version, based on a structured interview, was with five 12-year old children ( 2 boys, 3 girls) from a conveniently located school in the Gampaha District. They were interviewed to determine the difficulty level of the items, ease of understanding of concepts in the items, any discomfort when responding, and the appropriate length of the instrument. With this information the instrument was revised and pre-tested again on second group of ten 12year old children ( 4 boys, 6 girls) by a focus group discussion. Feedback was solicited on the difficulty level of its items, clarity of written instructions and response alternatives, and the instrument's formatting style. The feedback was used to further revise the instrument.

${ }^{1}$ Department of Psychological Medicine, ${ }^{2}$ Department of Community Medicine, Faculty of Medicine, University of Colombo; ${ }^{3}$ School of Social Work and Applied Human Sciences, Faculty of Social and Behavioural Sciences, University of Queensland, Brisbane, Australia 4072.

Correspondence: P de Z, e-mail: <ptdz@sltnet.lk> (Competing interests: none declared). Received 14 July 2004 and revised version accepted 15 November 2004. 


\section{Validity}

Consensual and content validity was determined using a Delphi Process [11], where six experts from the social and behavioural sciences were the judges. Since reliance only on back translation [10] in translating an instrument is not sufficient [12], consensual validation [11] was also used to determine the appropriateness of words used in the Sinhala version.

For consensual validity, the judges rated for each item, on a scale of 0 (total disagreement) to 9 (total agreement) - (i) if its conceptual meaning was retained after translating to Sinhala, (ii) if it was appropriate to be used with 12-year old Sinhala speaking schoolchildren, and (iii) if it was culturally relevant to Sri Lanka. For content validity the judges rated, again on a scale of 0 to 9-(i) if each item was an appropriate indicator of its scale or sub-scale, and (ii) if the composite of items was adequate to measure the concept assessed by a scale or sub-scale. Both the original English and the Sinhala versions were presented for validation. These ratings were summarised and presented for a second round of the Delphi Process. The repeat ratings were assessed for the degree of consensus.

\section{Reliability}

Reliability was determined by test-retest (inter-class correlation coefficient-ICC) and internal consistency (Chronbach's alpha). Test-retest reliability was assessed following a 14-day period and internal consistency computed on the second administration's data.

Reliability was assessed by (i) a large group, where the instrument was administered to the entire class (about 40 children), and (ii) a small group, where the instrument was administered to groups of (about) 20 children. All Grade 7 children from three conveniently located schools in the Gampaha District took part in the study. Those absent on the second day were not included in the analysis. The large group method totalled 119 children (61 boys, 58 girls). Eighteen gave incomplete instruments. The small group method totalled 111 children ( 54 boys, 57 girls). All returned completed instruments.

Children were assured of confidentiality and anonymity. It was explained that there was no direct benefit to them from the study and the information provided would be useful when planning services for Sri Lankan children. The concerned Ministry gave approval for the study. Ethical committee approval was obtained from the Faculty of Medicine, Colombo. Verbal consent of the participants was obtained.

\section{Results}

Based on the Delphi Process, items for the CTSPC's Sinhala version were chosen on the basis of the following criteria: (i) if $70 \%$ or more of the re-ratings were in category $0-3$, that item or scale or sub-scale was omitted, or reworded to make it acceptable. If reworded, the Delphi Process was repeated for that item or scale or sub-scale, and (ii) if $70 \%$ or more of the re-ratings were in categories 4-6 and 7-9 (summatively), that item or scale or subscale was retained.

The CTSPC's Sinhala version showed good consensual validity, except for one item, "sent you to your room". This item was omitted from the instrument as it was considered culturally inappropriate.

Based on the pre-tests and content validation, five items were added to the Sinhala version. They were "pulled the ear", "hit the head with the knuckles", "pulled the hair", "compared to a child whom parents consider as good and listed out his or her faults", and, "listed out child's faults in front of others in a way that made him or her feel ashamed". Further, five items in the original CTSPC were modified to make them culturally appropriate to be used in Sri Lanka (Table 1).

The test-retest reliabilities and internal consistencies for the full instrument, its scales and sub-scales were determined by way of a large and a small group administration. The results in the small group were superior to that of the large group (Tables 2 and 3).

Table 1. Items in the original CTSPC modified to make it representative to the Sri Lankan culture

\begin{tabular}{|c|c|c|c|}
\hline & Item in the original CTSPC & & Modified version in the CTSPC's Sinhala version \\
\hline 1. & $\begin{array}{l}\text { Hit you on the bottom with something like a belt, } \\
\text { hair brush, a stick or some other hard object }\end{array}$ & 1. & $\begin{array}{l}\text { Hit you on the bottom with something like the handle of a } \\
\text { big spoon, broom, cane, a stick or some other hard object }\end{array}$ \\
\hline 2. & Shouted, yelled or screamed at you & 2. & Shouted at you in a loud voice \\
\hline 3. & Swore or cursed at you & 3. & Scolded/cursed you in bad words \\
\hline 4. & $\begin{array}{l}\text { Hit you on some other part of the body besides the } \\
\text { bottom with something like a belt, hair brush, a stick } \\
\text { or some other hard object }\end{array}$ & 4. & $\begin{array}{l}\text { Hit you on some other part of the body besides the bottom } \\
\text { with something like a handle of a big spoon, broom, } \\
\text { cane, a stick or some other hard object }\end{array}$ \\
\hline 5. & Threatened you with a knife or gun & 5. & Threatened you with an axe, pestle or knife \\
\hline
\end{tabular}

CTSPC-Parent-Child Conflict Tactics Scale 
Table 2. Test-retest reliability results for the large and small group administrations

\begin{tabular}{lcc}
\hline & $\begin{array}{c}\text { Inter-class correlation } \\
\text { coefficient large group } \\
\text { (Sinhala version) }\end{array}$ & $\begin{array}{c}\text { Inter-class coefficient- } \\
\text { small group correlation } \\
\text { (Sinhala version) }\end{array}$ \\
\hline Full scale & $0.62^{*}$ & $0.88^{*}$ \\
Non-violent discipline scale & $0.64^{*}$ & $0.81^{*}$ \\
Psychological aggression scale & $0.69^{*}$ & $0.72^{*}$ \\
Corporal punishment scale & $0.47^{*}$ & $0.82^{*}$ \\
Severe assault sub-scale** & $0.35^{*}$ & $0.83^{*}$ \\
Very severe assault sub-scale** & 0.13 & $0.79^{*}$ \\
\hline
\end{tabular}

* Value significant at $\mathrm{p}<001$ level

** Severe assault and very severe assault sub-scales combine to form the physical abuse scale

Table 3. Internal consistency results for the original CTSPC and the present study's large and small group administrations

\begin{tabular}{lccc}
\hline & $\begin{array}{c}\text { Cronbach's alpha } \\
\text {-original (USA } \\
\text { version) }\end{array}$ & $\begin{array}{c}\text { Cronbach's alpha } \\
\text {-large group } \\
\text { (Sinhala version) }\end{array}$ & $\begin{array}{c}\text { Cronbach's group (Sinhala Version) } \\
\text { alpha-small }\end{array}$ \\
\hline Full scale & - & 0.81 & 0.95 \\
Non-violent discipline scale & 0.70 & 0.72 & 0.62 \\
Psychological aggression scale & 0.60 & 0.63 & 0.81 \\
Corporal punishment scale & - & 0.80 & 0.89 \\
Severe assault sub-scale* & -0.02 & 0.39 & 0.77 \\
Very severe assault sub-scale* & - & 0.80 & 0.82 \\
\hline
\end{tabular}

* Severe assault and very severe assault sub-scales combine to form the physical abuse scale

CTSPC-Parent-Child Conflict Tactics Scale

\section{Discussion}

The purpose of this study was to validate the CTSPC in Sri Lanka. Translating the CTSPC to Sinhala posed certain difficulties. Such difficulties in translating have been highlighted by the World Health Organisation [13]. To minimise these problems, the study involved a panel of judges rather than a single translator [14]. Validity of the instrument was determined by the Delphi Process [11]. The CTSPC's Sinhala version showed sufficient consensual and content validity, subject to certain deletions, additions and modifications.

The test-retest reliability and internal consistency were determined by a large and a small group administration. Though the CTSPC was originally developed for use in a classroom [7], when it was used so in Sri Lanka (i.e. large group), the results were not encouraging. Hence, it was administered in a small group, hypothesising that with the children having an opportunity to be in close contact with the instrument administrator and with greater opportunity to clarify doubts, the information obtained would be more reliable. The reliability for the full instrument, its scales and sub-scales were superior in the small than in the large group. A "rule of thumb" acceptability of test-retest reliability is an ICC value of at least 0.70 and an internal consistency alpha value of at least 0.60 [15]. All reported ICC and alpha values for the small group were above these acceptable values, indicating that the CTSPC's Sinhala version, in the small group, have good test-retest reliability and internal consistency.

The Sinhala versions' internal consistencies were superior to those of its original version (Table 3). This may indicate Sri Lankan children's willingness to report disciplinary strategies more than the children from the USA. This willingness may be because, unlike in the USA, punitive disciplinary strategies are generally considered normative in Sri Lanka and are not associated with shame or fear when reporting.

The CTSPC's Sinhala version has shown encouraging psychometric properties. It can be used with confidence in research, clinical screening of child maltreatment, and for evaluating preventive intervention programmes for maltreated children. A limitation of this study is that the instrument was validated only on 12-year old Sinhala speaking schoolchildren. Future research would need to validate the CTSPC to other groups in Sri Lanka.

\section{References}

1. De Silva DGH. Child abuse in Sri Lanka. In: SchwartzKenney B, McCauley M, Epstein MA, eds. Child Abuse: A Global View. Westport: Greenwood Press, 2001: 223-40.

2. Gershoff ET. Corporal punishment by parents and associated child behaviors and experiences: a metaanalysis and theoretical review. Psychological Bulletin 2002; 128: 539-79. 
3. Mrazek PJ, Haggerty RJ. Reducing Risks for Disorders: Frontliners for Preventive Intervention. Washington DC: National Academy Press, 1994: 123-31.

4. Rohner RP, Bourque SL, Elordi CA. Childrens' perceptions of corporal punishment, caretaker acceptance, and psychological adjustment in a poor, biracial Southern community. Journal of Marriage and the Family 1996; 58: $842-52$.

5. Sanders B, Becker-Lausen E. The measurement of psychological maltreatment: Early data on the child abuse and trauma scale. Child Abuse and Neglect 1995; 19: 315-23.

6. Bernstein DP, Fink L, Handelsman L, Foote J, Lovejoy $\mathrm{M}$, Wenzel $\mathrm{K}$ et al. Initial reliability and validity of a new retrospective measure of child abuse and neglect. American Journal of Psychiatry 1994; 151: 1132-6.

7. Straus MA, Hamby SL, Finklehor D, Moore DW, Runyan D. Identification of child maltreatment with the parentchild conflict tactics scales: Development and psychometric data for a national sample of American parents. Child Abuse and Neglect 1998; 22: 249-70.

8. Hui HC, Triandis HC. Measurement in cross-cultural psychology: A review and comparison of strategies. Journal of Cross-Cultural Psychology 1985; 16: 131-52.
9. Hamby SL, Finkelhor D. Choosing and using child victimisation questionnaires. Juvenile Justice Bulletin 2001; 9: 13-6.

10. Neuman WL. Social Research Methods: Qualitative and Quantitative Approaches. 3rd ed. Boston, MA: Viacom Company, 1997.

11. Jones J, Hunter D. Consensus methods for medical and health services research. British Medical Journal 1995; 311: $376-80$.

12. Sumathipala A, Murray J. New approach to translating instruments for cross-cultural research: A combined qualitative and quantitative approach for translation and consensus generation. International Journal of Methods in Psychiatric Research 2000; 9: 87-95.

13. World Health Organization. International Classification of Impairments, Disabilities and Handicap (ICIDH). Field Trial Protocol for Linguistic Analysis. Geneva: World Health Organization, 1997; 7-11.

14. Guilliman F, Bombardier C, Beaton D. Cross-cultural adaptation of health related quality of life measures: Literature review of proposed guidelines. Journal of Clinical Epidemiology 1993; 46: 1417-32.

15. De Vaus DA. Surveys in Social Research. 3rd ed. Australia: Allen \& Unwin Pty Ltd., 1991. 